\title{
The effect of the antisaccade task on microsaccade suppression in the Posner cueing paradigm
}

Sofia Krasovskaya 1,2 (Krasov.sofia@gmail.com), Árni Kristjansson 1,3, Joseph MacInnes 1,2

1 - National Research University 'Higher School of Economics', 2 - Vision Modelling Lab, 3 - Icelandic Vision Lab

What is the functional purpose of microsaccades?

To investigate the functional purpose of microsaccadic eye movements (MS), we studied MS during the antisaccade task

- We compared participants' performance across 3 conditions:
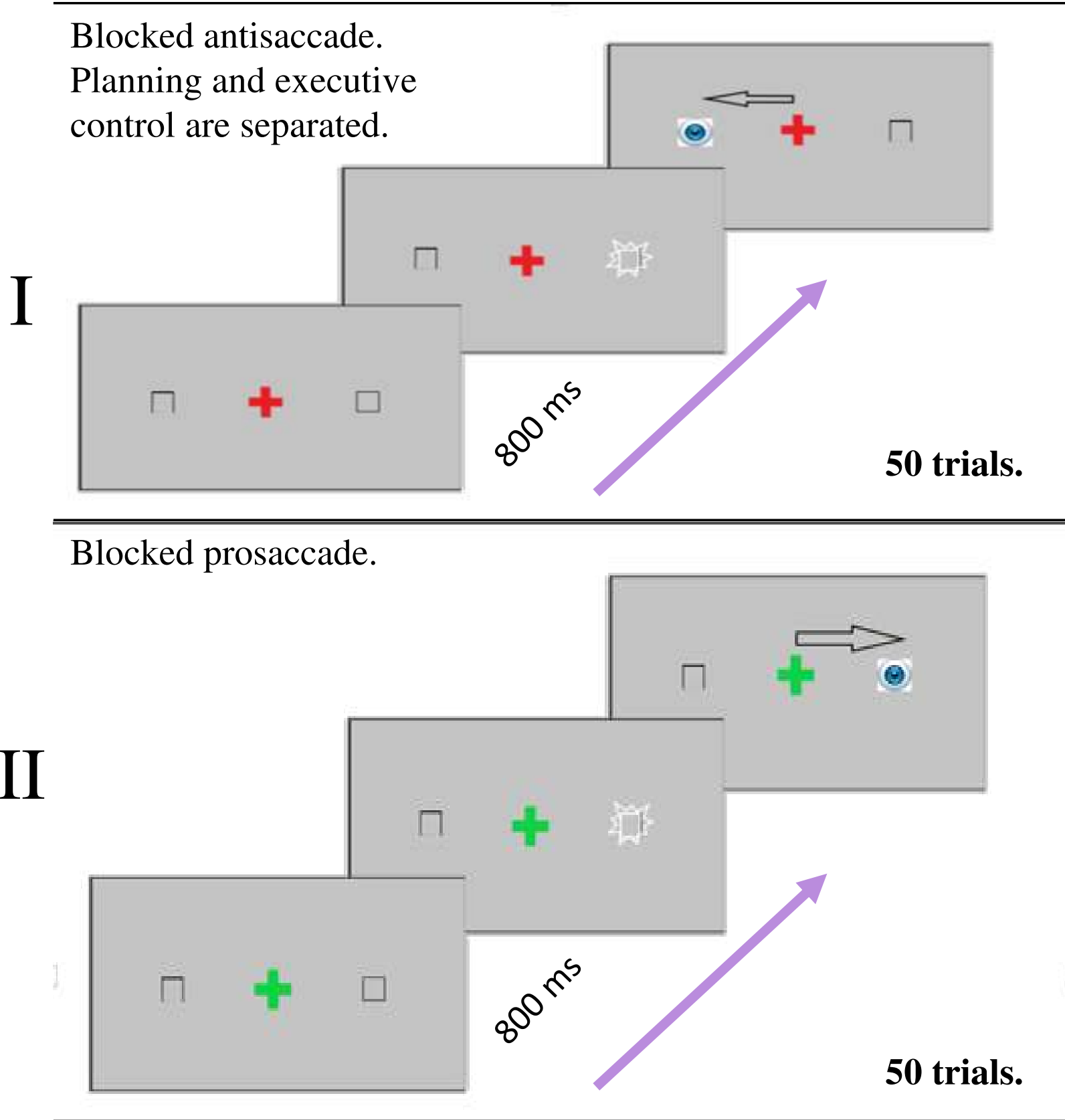

III Randomly mixed I + II

200 trials

\section{HYPOTHESIS}

The blocked antisaccade condition will lead to fewer MS as compared to saccade trials.

\section{METHODS}

20 participants contributing to all 3 conditions

Eyelink 1000

MS detection algorithm from open source software in $\mathbf{R}$

(Engbert et al., 2015)

Calculation of MS in the pre-cue condition (Figure 1).

\section{RESULTS}

- Lower occurrence of MS during the blocked trials as compared to the mixed condition. We believe that in the mixed trials participants are in an attentional state of uncertainty, which results in more microsaccade corrections.

- Main effect of block type $(\mathrm{P}=2.795 \mathrm{e}-09)$, though these two effects did not interact $(\mathrm{P}=0.78)$.

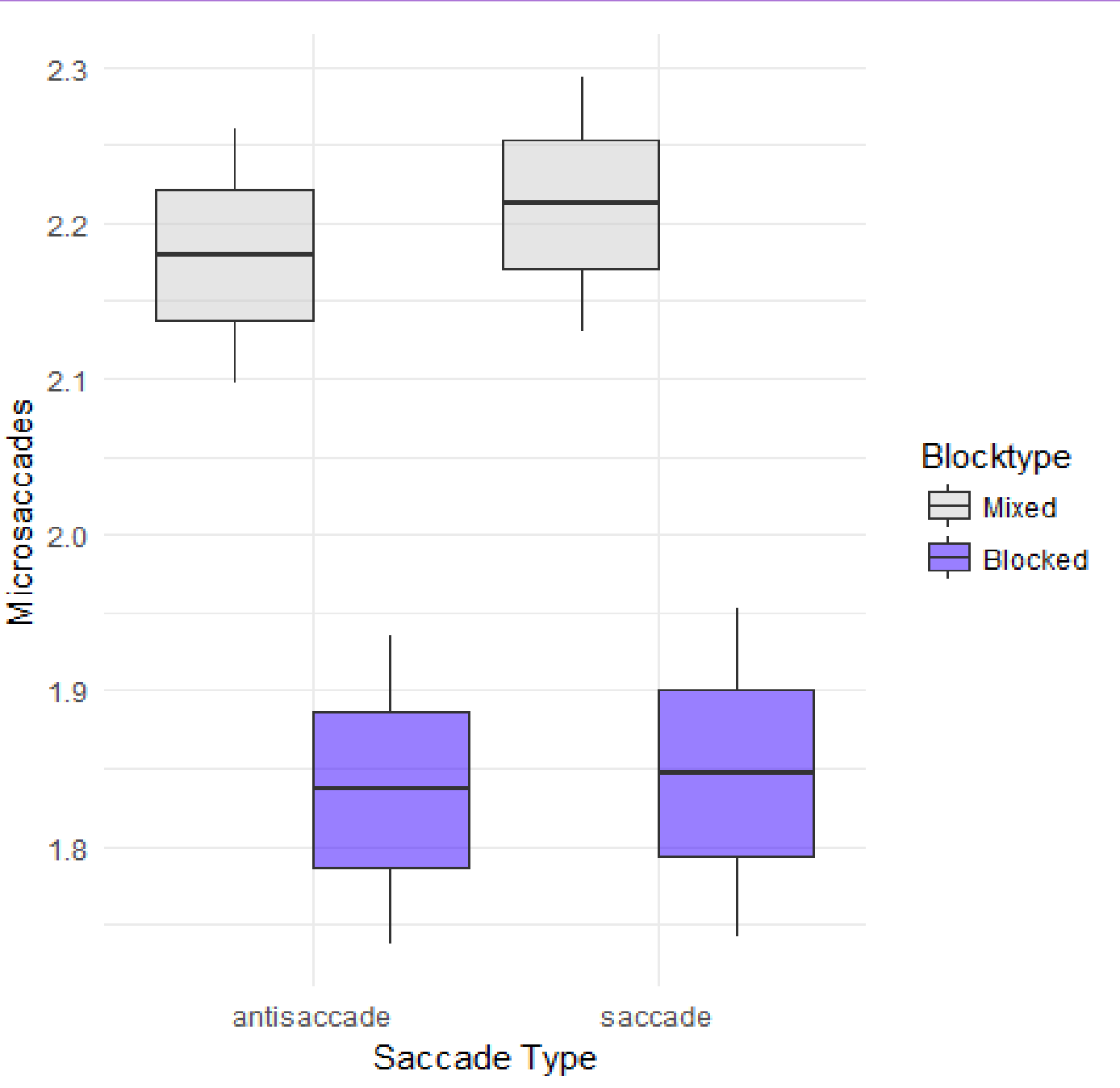

Figure 1. The amount of microsaccades per saccade type (saccade versus antisaccade) and per block type (blocked versus
Large difference in latency between antisaccades and prosaccades (Figure 2) with an interaction between saccade and block types $(\mathrm{P}=0.0001595)$.

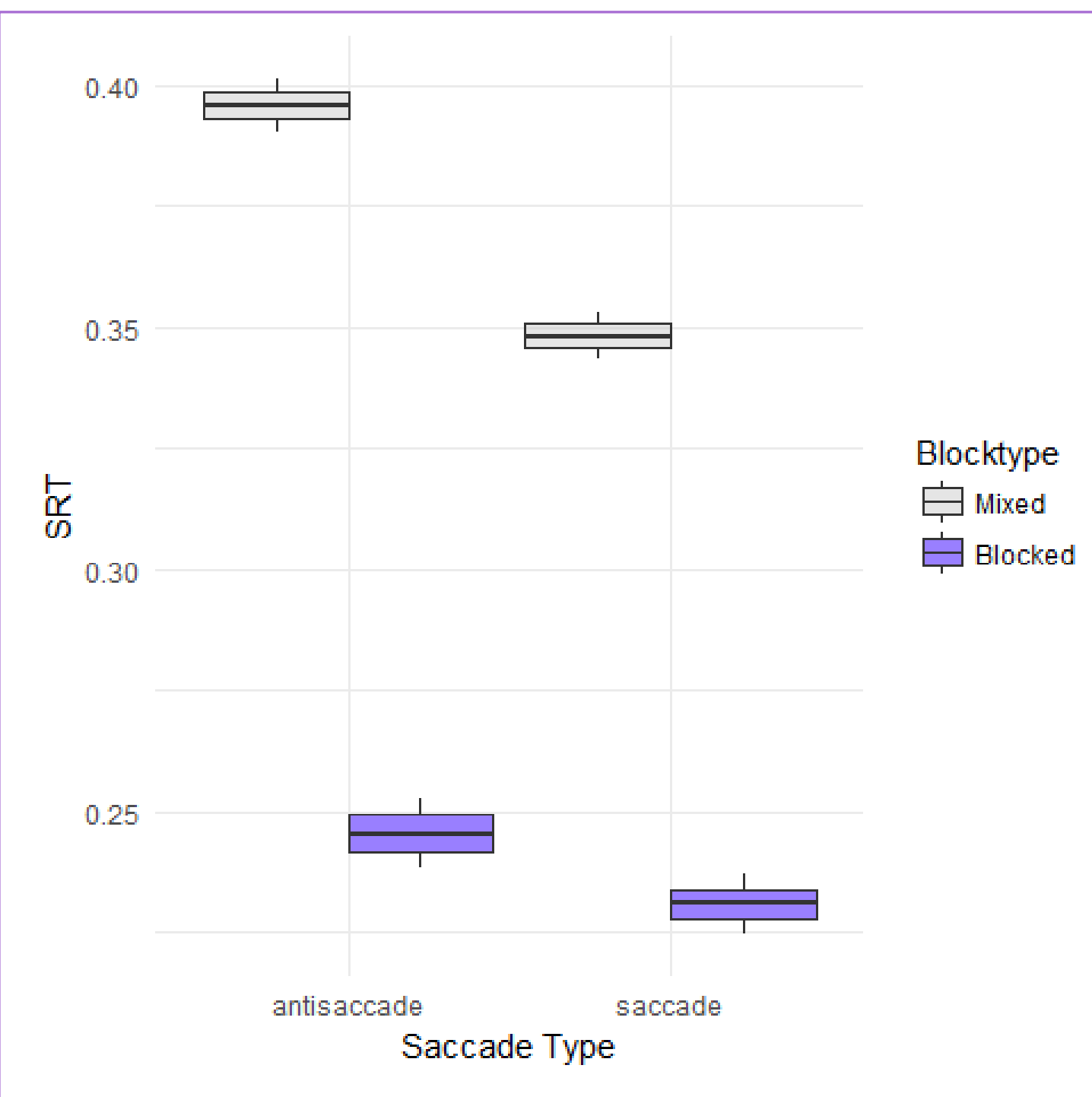

Figure 2. Mean RT in antisaccade versus prosaccade trials.

\section{CONCLUSION}

Suppressed MS rates in the blocked trials, though we did not find a significant effect of saccade type yet

- Interaction in RT between antisaccade and task switching, both of which are thought to require executive control

- The next steps would be to analyze and compare MS execution during the pre-cue fixation duration across the 3 conditions.

References
Engbert, R., \& Kliegl, R. (2003). Microsaccades uncover the orientation of covert attention. Vision Research, 43(9), 1035-1045.

Engbert, R., Sinn, P., Mergenthaler, K., \& Trukenbrod, H. (2015) Microsaccade Toolbox

Posner, M. I. (1980). Orienting of attention. Quarterly journal experimental psychology, 32(1), 3-25. 